\title{
KRAS(G12D) can be targeted by potent inhibitors via formation of salt bridge
}

\author{
Zhongwei Mao ${ }^{1}$, Hongying Xiao ${ }^{1,2}$, Panpan Shen ${ }^{3}$, Yu Yang ${ }^{3}$, Jing Xue ${ }^{1}$, Yunyun Yang ${ }^{1}$, Yanguo Shang ${ }^{1}$, Lilan Zhang ${ }^{3}$, \\ Xin $\mathrm{Li}^{1,2}$, Yuying Zhang ${ }^{4}$, Yanan Du${ }^{4}$, Chun-Chi Chen ${ }^{3}$, Rey-Ting Guo $\mathbb{0}^{3 凶}$ and Yonghui Zhang ${ }^{1 凶}$
}

\begin{abstract}
KRAS mutation occurs in nearly 30\% of human cancers, yet the most prevalent and oncogenic KRAS(G12D) variant still lacks inhibitors. Herein, we designed a series of potent inhibitors that can form a salt bridge with KRAS's Asp12 residue. Our ITC results show that these inhibitors have similar binding affinity with both GDP-bound and GTP-bound KRAS (G12D), and our crystallographic studies reveal the structural basis of inhibitor binding-induced switch-ll pocket in KRAS(G12D), experimentally confirming the formation of a salt bridge between the piperazine moiety of the inhibitors and the Asp12 residue of the mutant protein. Among KRAS family proteins and mutants, both ITC and enzymatic assays demonstrate the selectivity of the inhibitors for KRAS(G12D); and the inhibitors disrupt the KRAS-CRAF interaction. We also observed the inhibition of cancer cell proliferation as well as MAPK signaling by a representative inhibitor (TH-Z835). However, since the inhibition was not fully dependent on KRAS mutation status, it is possible that our inhibitors may have off-target effects via targeting non-KRAS small GTPases. Experiments with mouse xenograft models of pancreatic cancer showed that TH-Z835 significantly reduced tumor volume and synergized with an antiPD-1 antibody. Collectively, our study demonstrates proof-of-concept for a strategy based on salt-bridge and inducedfit pocket formation for KRAS(G12D) targeting, which warrants future medicinal chemistry efforts for optimal efficacy and minimized off-target effects.
\end{abstract}

\section{Introduction}

The oncogenic impacts of the KRAS gene were first reported in 1980s, making KRAS one of the first identified oncogenes $^{1}$. It is known that KRAS protein functions as a molecular switch: it responds to upstream EGFR activation and regulates the downstream MAPK and PI3K/ mTOR pathways, eventually controlling cell proliferation, differentiation, and survival ${ }^{2-5}$.

Clinical data have implicated the driver mutations of the KRAS residue Gly12 (G12), and basic studies have shown

\footnotetext{
Correspondence: Rey-Ting Guo (guoreyting@hubu.edu.cn) or

Yonghui Zhang (zhangyonghui@tsinghua.edu.cn)

${ }^{1}$ School of Pharmaceutical Science, Tsinghua-Peking Center for Life Sciences,

Tsinghua University; Beijing Advanced Innovation Center for Human Brain Protection, Beijing, China

${ }^{2}$ Joint Graduate Program of Peking-Tsinghua-NIBS, School of Life Sciences, Tsinghua University, Beijing, China

Full list of author information is available at the end of the article

These authors contributed equally: Zhongwei Mao, Hongying Xiao, Panpan

Shen, Yu Yang.
}

that such mutations impair both this enzyme's intrinsic and GTPase-activating protein (GAP)-stimulated GTP hydrolysis activity, ${ }^{6,7}$, promoting oncogenesis. Despite nearly four decades of efforts, no direct KRAS inhibitor has been approved for medical use. There is consensus that the difficulty in developing direct KRAS inhibitors relates on the one hand to the picomolar affinity of GTP and GDP to KRAS (the intracellular concentrations of these metabolites are much higher), and on the other hand to an absence of suitable deep pockets for allosteric regulation.

One major breakthrough for KRAS inhibition was the discovery of an allosteric switch-II pocket (S-IIP) that is induced by covalent inhibitors of KRAS bearing the G12C driver mutation ${ }^{8}$. Studies have shown that induction of S-IIP results from covalent bond formation between the electrophilic acryloyl moieties of these inhibitors and the nucleophilic thiol moiety of the Cys residue at position $12^{9-16}$. These KRAS(G12C) inhibitors have shown

\section{c The Author(s) 2022}

\footnotetext{
(c) Open Access This article is licensed under a Creative Commons Attribution 4.0 International License, which permits use, sharing, adaptation, distribution and reproduction cc) in any medium or format, as long as you give appropriate credit to the original author(s) and the source, provide a link to the Creative Commons license, and indicate if changes were made. The images or other third party material in this article are included in the article's Creative Commons license, unless indicated otherwise in a credit line to the material. If material is not included in the article's Creative Commons license and your intended use is not permitted by statutory regulation or exceeds the permitted use, you will need to obtain permission directly from the copyright holder. To view a copy of this license, visit http://creativecommons.org/licenses/by/4.0/.
} 
promising results in recent clinical trials, although it is notable that they exclusively target KRAS in inactive state $^{9}$. Despite these progresses with inhibitors of KRAS (G12C), the most prevalent and oncogenic G12 mutant variant is $\mathrm{KRAS}(\mathrm{G} 12 \mathrm{D})$, which is estimated to impact more than 50\% patients with pancreatic ductal adenocarcinoma ${ }^{17}$.

Various strategies have been testified for targeting KRAS(G12D), including those using indole-based small molecules to target a switch-I/II pocket, a compound (KAL-21404358) to target the P110 site, a pan-RAS inhibitor (compound 3144) to target the A59 site, and a cyclic peptide (KD2) to target KRAS(G12D) ${ }^{18-21}$. However, none of these molecules targets KRAS(G12D) with suitably low (micromolar) concentration. We speculated that a compound targeting the aspartic acid residue of KRAS(G12D) may somehow bind to KRAS(G12D) (similar to that formed between inhibitors and the cysteine residue of KRAS(G12C)), as a result may induce an allosteric pocket. This would perhaps enable pharmacological inhibition of this more prevalent oncodrivermutation-bearing KRAS variant. We successfully developed a series of small molecule inhibitors of KRAS (G12D), which function by inducing an allosteric S-IIP and forming a salt bridge bonding with Asp12 residue, as confirmed by crystallographic studies. These inhibitors bind to both GDP-bound and GTP-bound KRAS(G12D), efficiently disrupt KRAS-CRAF interaction, but do not bind to wide type and G12C mutant KRAS. Our studies showed that they disrupted MAPK signaling, reduced tumor volume, and synergized with an anti-PD1 antibody in mouse xenograft models of pancreatic cancer. However, these inhibitors are also associated with off-target effects, likely due to binding and inhibiting some non-KRAS small GTPases, which warrants further structural optimizations.

\section{Results}

A salt bridge-based strategy for targeting KRAS(G12D) with a methyl-substituted piperazine inhibitor

Assuming that the $\alpha$-carboxylic acid moiety of Asp12 is deprotonated under physiological conditions, we pursued a strategy based on the formation of a strong interaction (salt-bridge) between Asp12 and an alkyl amine moiety on an inhibitor. As the overall structures of the KRAS(G12C) and KRAS(G12D) variants are highly similar, we started our experiment based on a scaffold of the G12C inhibitor MRTX $^{22}$ (Supplementary Fig. S1a). Removal of MRTX's acryloyl moiety exposed a piperazine moiety that was predicted to position this alkyl amine in close enough proximity to Asp12 (2.2 $\AA$ ) to support salt-bridge interaction (Supplementary Fig. S1b).

Pursuing this, we synthesized TH-Z801 (Fig. 1a; Supplementary Fig. S2), which exerted relatively weak inhibition $\left(\mathrm{IC}_{50}=115 \mu \mathrm{M}\right)$, assessed based on the GDP/ GDP exchange rate of KRAS(G12D) as catalyzed by SOS ${ }^{9}$. Additional structure-activity relationship (SAR) studies showed that replacement of the piperazine moiety with non-amine moieties dramatically decreased the inhibitory activity, supporting the functional contribution of a saltbridge interaction in slowing down the GDP/GDP exchange rate (Fig. 1a; Supplementary Fig. S2).

Further chemical exploration focusing on piperazine substitution yielded TH-Z816 (wherein the piperazine was (R)-methyl-substituted), which had relatively strong inhibition activity $\left(\mathrm{IC}_{50}=14 \mu \mathrm{M}\right)($ Fig. 1a, b). We further conducted isothermal titration calorimetry (ITC) assays to test whether TH-Z816 can bind directly to KRAS (G12D). Indeed, the detected binding affinity $\left(\mathrm{K}_{\mathrm{D}}\right)$ of TH-Z816 with KRAS(G12D) (GDP-bound) was $25.8 \mu \mathrm{M}$ (Fig. 1c).

\section{A complex structure revealed an induced-fit pocket and confirmed the salt-bridge interaction}

We solved a $2.13 \AA$ co-crystal structure of KRAS(G12D) in complex with TH-Z816 (Supplementary Table S1). Our structure showed that TH-Z816 induced an allosteric pocket positioned under the KRAS(G12D) switch-II region (Fig. 1d); no such pocket was evident in the inhibitor-free structure (Supplementary Fig. S1c). This induced-fit pocket was located near the $\alpha 2$-helix, switchII, $\alpha 3$-helix, the P-loop, and the central $\beta$-sheet of KRAS (Fig. 1d). Note that the pocket shape was quite similar (RMSD $0.293 \AA$, 148 to 148 atoms) to the MRTX-induced SII-P of KRAS(G12C) (Supplementary Fig. S1d).

Well-defined electron density clearly supported the conformation of TH-Z816 and KRAS(G12D) (Fig. 1e). Specifically, the naphthyl moiety of TH-Z816 embedded deeply into the pocket and formed hydrophobic interactions with residues Val9, Met72, Phe78, Gln99, Ile100, and Val103 (Supplementary Fig. S1e). There are also four pairs of polar interactions between TH-Z816 and residues His95, Glu62, Gly60, and Asp12 (Fig. 1f). Note that an ionic bond and a hydrogen bond together comprise the anticipated salt-bridge: on one hand, the positively charged piperazine moiety of TH-Z816 and negatively charged side chain of Asp12 are close enough to support an ionic bond (Fig. 1g); on the other hand, the measured bond angle $\left(166.7^{\circ}\right)$ and length $(2.7 \AA)$ supports the presence of a hydrogen bond between these two moieties (Fig. 1g).

\section{A cyclization strategy to improve inhibitory potency by optimizing $\Delta S$}

Further optimization of TH-Z816 was guided by the following thermodynamic rule, $\Delta G=\Delta H-T \Delta S^{23,24}$. We sought to optimize $\Delta S$ by restraining the conformational freedom of the piperazine moiety. Given the axial position 


\section{a}

$\begin{array}{cccc}\text { a } & \text { TH-Z801 } & \text { TH-Z814 } & \text { TH-Z816 } \\ \mathrm{IC}_{50} & 115 \mu \mathrm{M} & 330 \mu \mathrm{M} & 14 \mu \mathrm{M}\end{array}$<smiles>CN1CCN(C)CC1</smiles><smiles></smiles>

c

[TH-Z816] $800 \mu \mathrm{M}$
[KRAS G12D (GDP-

[KRAS G12D (GDP-bound)] $26 \mu \mathrm{M}$

$K_{\mathrm{D}} 25.8 \pm 5.78 \mu \mathrm{M}$

$\mathrm{N} 0.709 \pm 0.139$

$\Delta H-17.7 \pm 4.88 \mathrm{kcal} / \mathrm{mol}$
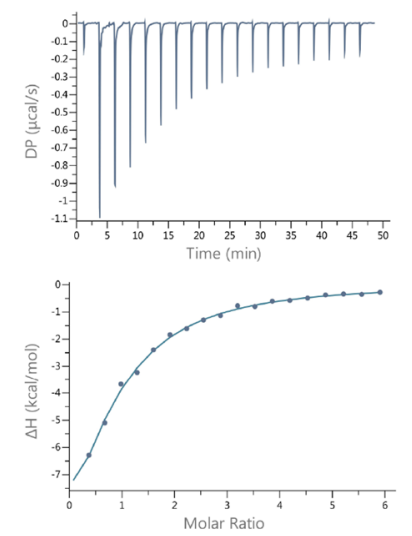

b

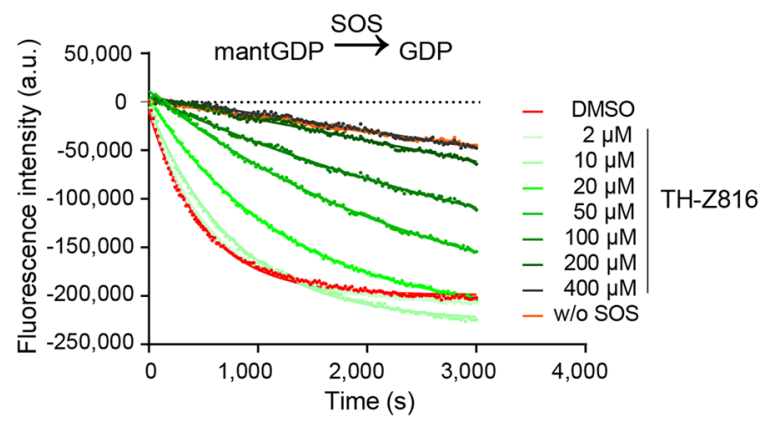

d

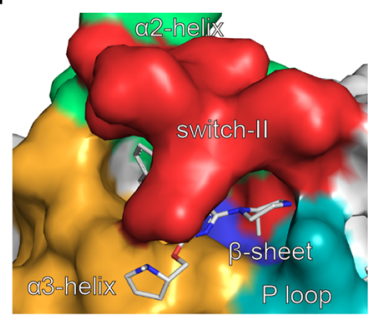

e

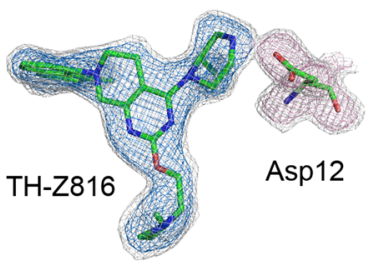

g

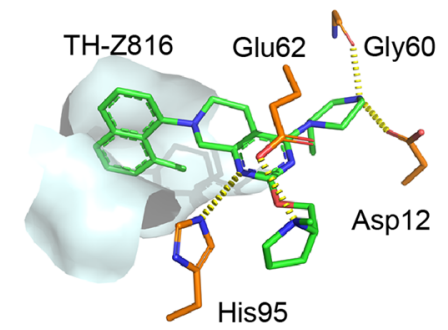

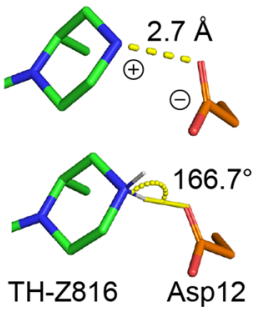

Fig. 1 A piperazine-focused strategy for targeting KRAS(G12D) via salt-bridge interaction. a Chemical structures of TH-Z801 and TH-Z816 with exposed piperazines, structure of TH-Z814 with non-amine moiety. $\mathbf{b}$ Inhibitory activities of TH-Z816 on KRAS(G12D) measured by SOS-catalyzed

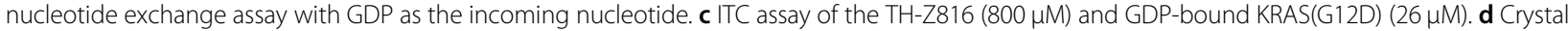
structure (PDB ID: 7EW9) of KRAS(G12D) bound to TH-Z816 (white stick). The binding pocket is formed by the a2-helix (green), switch-II (red), a3-helix (orange), P-loop (teal), and the central $\beta$-sheet (blue) of KRAS(G12D). e Fo-Fc maps of TH-Z816 (blue mesh, 2.5 ; ; gray mesh, $1.5 \sigma$ ) and Asp12 (pink

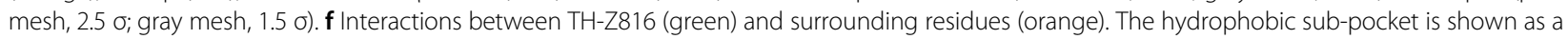
surface diagram. $\mathbf{g}$ The ionic bond (upper panel) and hydrogen bond (lower panel) between piperazine and Asp12.

of the methyl group, we employed a cyclization strategy based on TH-Z816 and generated the bicyclic compound TH-Z827 (Supplementary Fig. S3a). As TH-Z827 showed a more than eight-fold increase in potency $\left(\mathrm{IC}_{50}=\right.$ $3.1 \mu \mathrm{M})$, we subsequently adopted a $\Delta S$-focused cyclization strategy and designed additional bicyclic compounds (Fig. 2a), among which the most potent one was TH-Z835 $\left(\mathrm{IC}_{50}=1.6 \mu \mathrm{M}\right)$ (Fig. 2b).

We next conducted ITC assays to measure the binding affinity of these bicyclic compounds to KRAS(G12D) in the presence of GDP (Fig. 2c; Supplementary Fig. S3b, c). Compared with TH-Z816, bicyclic compounds TH-Z827, TH-Z835, and TH-Z837 had unfavorable $\Delta H$ changes yet favorable $\Delta S$ changes (Fig. 2d), indicating that the binding affinity $(\Delta G)$ increase of these bicyclic compounds can be attributed to $\Delta S$ improvement.

\section{G12D inhibitors bind to both GDP-bound and GMPPNP- bound KRAS with similar affinities}

Previous studies have shown that KRAS exists in cells in both GTP-bound and GDP-bound states ${ }^{13,25-30}$. A molecular docking analysis of our KRAS(G12D) inhibitors indicated that the absence of an acryloyl moiety in the inhibitors results in sufficient space $(4.7 \AA)$ for the $\gamma$ phosphate of GTP, while the salt-bridge interaction between Asp12 of KRAS and the piperazine moiety of our inhibitors is maintained (Supplementary Fig. S4a).

To reveal whether these inhibitors bind to the GTPbound KRAS(G12D), we first treated the purified protein with GMPPNP (a stable analog of GTP) and EDTA, following a well-established nucleotide-exchange protocol ${ }^{8,9}$. We next aimed at crystallizing GMPPNP-containing KRAS(G12D) in complex with these more active 

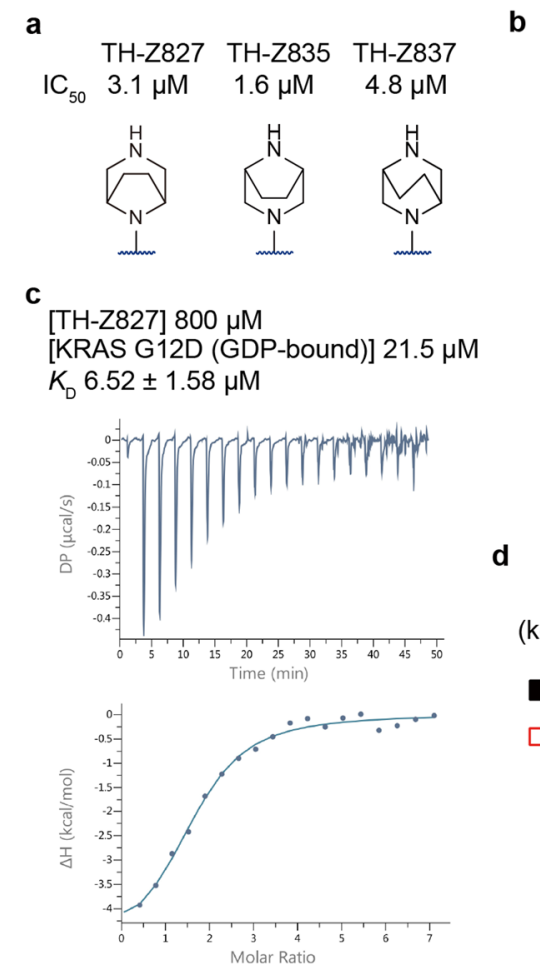

b

d
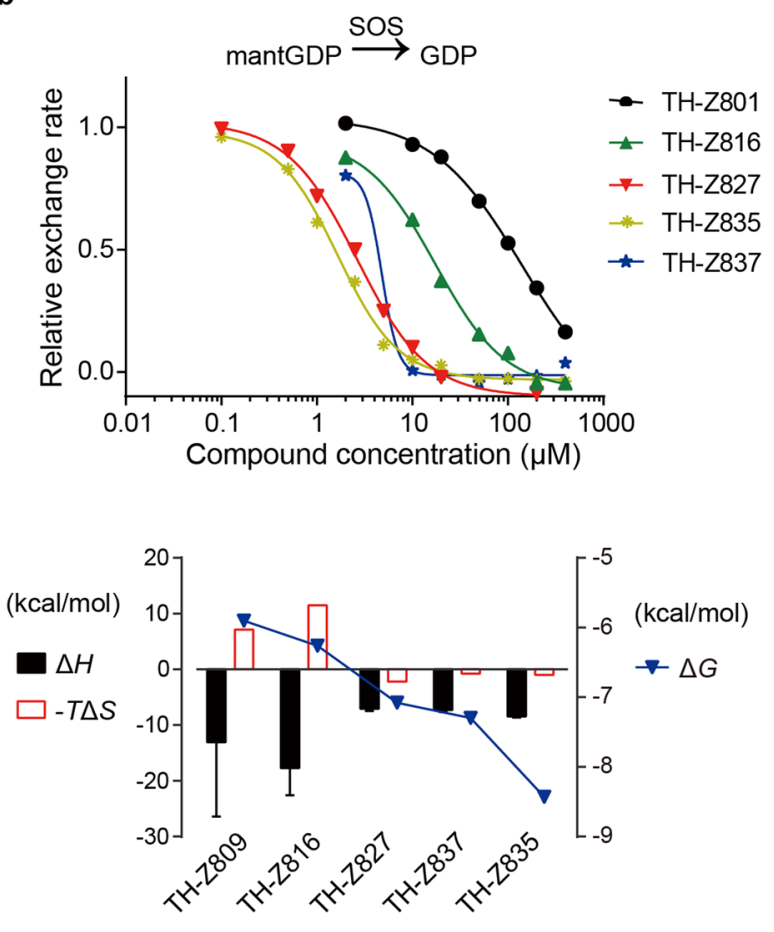

Fig. 2 A cyclization strategy to improve inhibitory activities and binding potency. a Chemical structures of bicyclic compounds TH-Z827, THZ835, and TH-Z837. b Inhibitory activities of these bicyclic compounds on KRAS(G12D) measured by SOS-catalyzed nucleotide exchange assay with GDP as the incoming nucleotide. $\mathbf{c}$ ITC assay of the TH-Z827 (800 $\mu \mathrm{M})$ and GDP-bound KRAS(G12D) (21.5 $\mu \mathrm{M})$. d $\Delta G, \Delta H$, and $\Delta S$ analysis of compounds tested by ITC assays. For each compound, $\Delta H$ and -T $\Delta S$ values are presented referring to the left axis, while the $\Delta G$ value is presented referring to the right axis.

inhibitors TH-Z827 and TH-Z835, and successfully solved a $2.25 \AA$ co-crystal structure of KRAS(G12D) in complex with TH-Z827 (PDB ID: 7EWA; Fig. 3a; Supplementary Table S1). This crystal structure of KRAS (G12D) was trimeric, with monomer A bound to GDP and TH-Z827, monomer B bound to GMPPNP and TH$\mathrm{Z} 827$, and monomer $\mathrm{C}$ bound to GMPPNP. We also solved a co-crystal structure of KRAS(G12D) in complex with TH-Z835 (PDB ID: 7EWB; Fig. 3a; Supplementary Table S1). In these two inhibitors-KRAS(G12D) structures, the electron densities were well-defined for inhibitors, Asp12, and both GMPPNP and GDP. Our data confirmed that both TH-Z827 and TH-Z835 are able to interact with Asp12 and form a salt bridge for both GDPbound and GMPPNP-bound KRAS(G12D) (Fig. 3a). It is noted that in the inhibitor-free GMPPNP-bound state, switch II more tightly bound to the $\gamma$-phosphate. However, the binding of TH-Z835 shifted the conformation of switch II towards the inhibitor-free GDP-bound conformation (analysis shown in Fig. 3b).

We next conducted ITC assays to measure the binding affinities between G12D inhibitors and GMPPNP-bound KRAS (G12D) (Supplementary Fig. S4b). The detected binding affinities for each of the tested compounds were within a narrow range for both the GDP-bound and the GMPPNP-bound forms (Supplementary Fig. S4c), and EDTA-catalyzed nucleotide exchange assays that test the GDP/GTP binding preference of KRAS(G12D) supported our ITC results, showing no GDP/GTP binding preference for KRAS(G12D) in the presence of our inhibitors (Fig. 3c). In contrast, KRAS(G12C) had a significantly lower affinity for GTP than for GDP in the presence of the G12C inhibitor MRTX (Supplementary Fig. S4d), which was consistent with the previous reports ${ }^{9}$. We also conducted SOS-catalyzed nucleotide exchange assays of KRAS(G12D), which experimentally confirmed that our inhibitor TH-Z835 does inhibit both mantGMPPNP/ GPPNP exchange and GPPNP/mantGMPPNP exchange (Fig. 3d; Supplementary Fig. S4e).

\section{Mutant selectivity of the KRAS(G12D) inhibitor TH-Z827}

We next studied the mutant selectivity of our G12D inhibitors using ITC assays and did not detect measurable binding for TH-Z827 with KRAS(WT) or with KRAS (G12C), no matter whether the proteins were GDP-bound or GMPPNP-bound (Fig. 4a; Supplementary Fig. S5). SOS-catalyzed nucleotide exchange assays also showed that TH-Z827 exerted a nearly 10 -fold stronger inhibition 
a

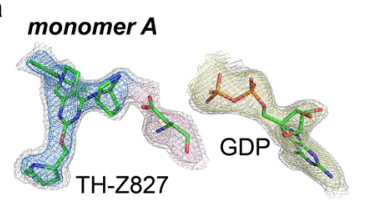

b
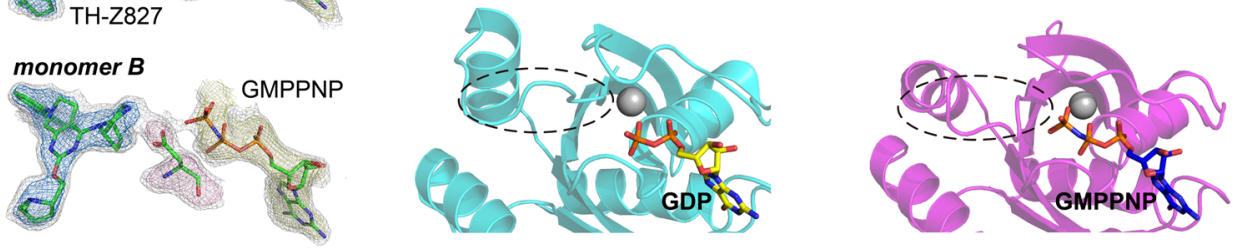

monomer A

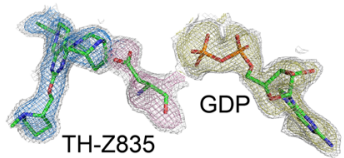

monomer $B$
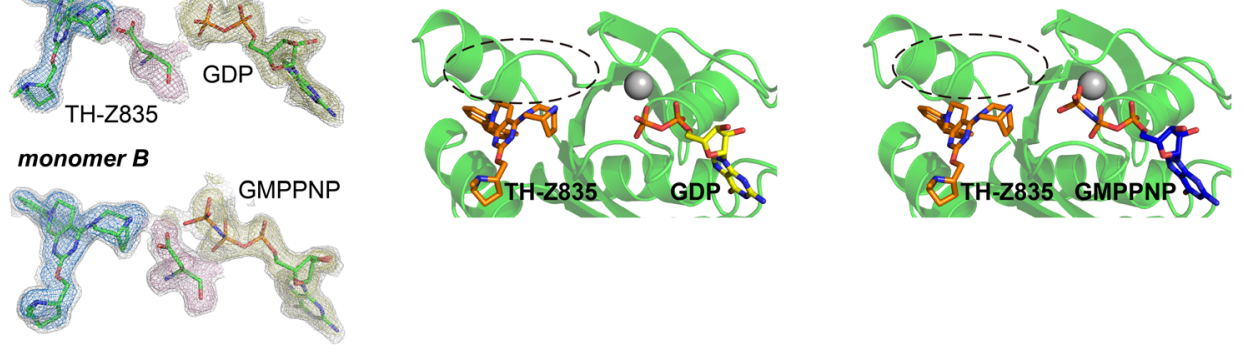

c

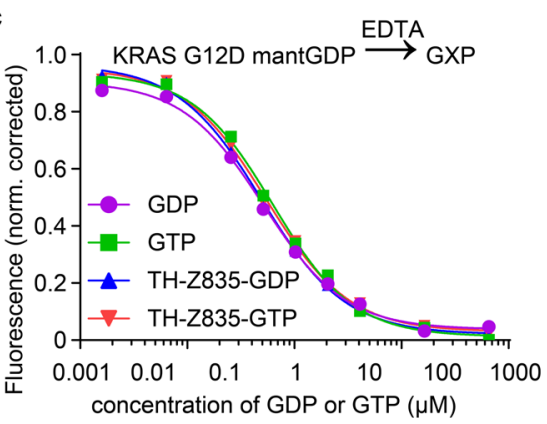

d

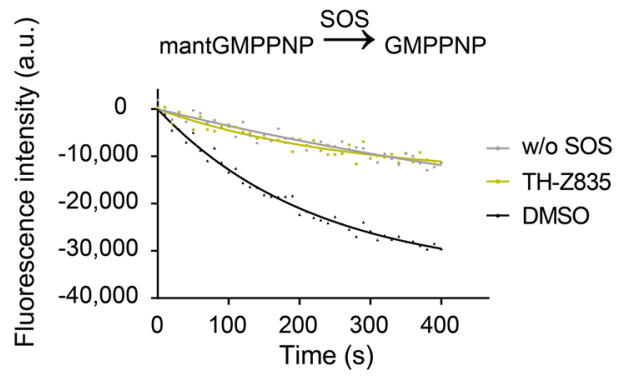

Fig. 3 G12D inhibitors bind to both GDP-bound and GMPPNP-bound KRAS. a Upper panel: Fo-FC map of TH-Z827, Asp12, and GDP (PDB ID: 7EWA, monomer A). Fo-Fc map of TH-Z827, Asp12, and the GTP analog GMPPNP (PDB ID: 7EWA, monomer B). Lower panel: Fo-FC map of TH-Z835, Asp12, and GDP (PDB ID: 7EWB, monomer A). Fo-Fc map of TH-Z835, Asp12, and GMPPNP (PDB ID: 7EWB, monomer B). In all graphs, the $1.5 \sigma$ Fo-FC maps of all shown elements are shown in gray mesh. The color scheme of $2.5 \sigma$ Fo-Fc maps is: blue for inhibitors, pink for Asp12, and yellow for nucleotides. b KRAS(G12D) conformational change analysis for drug-free GDP-bound protein (PDB: 4EPR), drug-free GMPPNP-bound protein (PDB: 5USJ), TH-Z835 and GDP-bound protein (PDB: 7EWB, chain A), and TH-Z835 and GMPPNP-bound protein (PDB: 7EWB, chain B). c EDTA-mediated competition between fluorescently labeled mantGDP loaded on KRAS and free nucleotide (GDP or GTP). The experiment was carried out with KRAS (G12D) alone or with KRAS(G12D) treated with TH-Z835. $\mathbf{d}$ Inhibitory activity of TH-Z835 measured by SOS-catalyzed nucleotide exchange assays with GMPPNP as the incoming nucleotide.

for KRAS(G12D) than for KRAS(G12C) $\left(\mathrm{IC}_{50}=2.4 \mu \mathrm{M}\right.$ vs $\left.\mathrm{IC}_{50}=20 \mu \mathrm{M}\right)($ Fig. $4 \mathrm{~b})$.

After confirming the mutant-selectivity of targeting KRAS, we further tested whether our inhibitor could selectively block the interactions of various KRAS mutants with its effector protein. CRAF is a well-studied KRAS effector protein, and the KRAS-CRAF interaction is known to promote MAPK signal transduction ${ }^{31-33}$. We established a split-luciferase reporter system using HEK 293T cells (Fig. 4c). Upon doxycycline treatment, the cells express both full-length KRAS (fused to N-luciferase) and a truncated CRAF variant (comprising residue 50-220) fused to C-luciferase. After lysis, the supernatant was incubated with our inhibitor and the substrate luciferin. When KRAS binds to CRAF, luciferase complementation results in the emission of a luminescence signal. The results showed that TH-Z827 blocked the KRAS (G12D)-CRAF interaction with an $\mathrm{IC}_{50}$ value of $42 \mu \mathrm{M}$ but did not affect CRAF's interaction with KRAS(WT) or KRAS(G12C) at this concentration (Fig. 4c).

\section{Inhibition of KRAS(G12D) mutant cell lines}

The promising results for the inhibitory activity and mutant-selectivity from the ITC and KRAS-CRAF 
a

\begin{tabular}{cc}
\multicolumn{2}{c}{ Binding affinities with $\mathrm{TH}-\mathrm{Z} 827$} \\
\hline KRAS WT-GDP & No binding \\
KRAS WT-GMPPNP & No binding \\
KRAS G12C-GDP & No binding \\
KRAS G12C-GMPPNP & No binding \\
KRAS G12D-GDP & $6.52 \mu \mathrm{M}$ \\
KRAS G12D-GMPPNP & $7.01 \mu \mathrm{M}$ \\
\hline
\end{tabular}

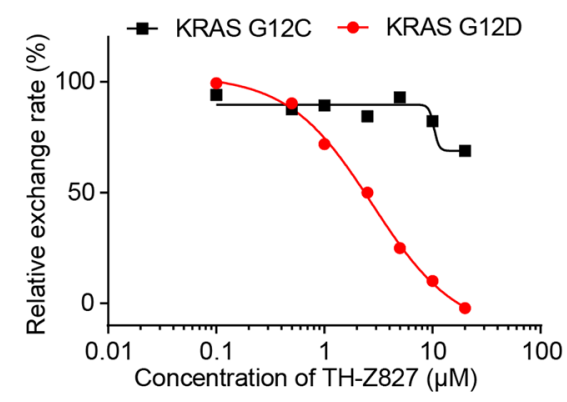

C

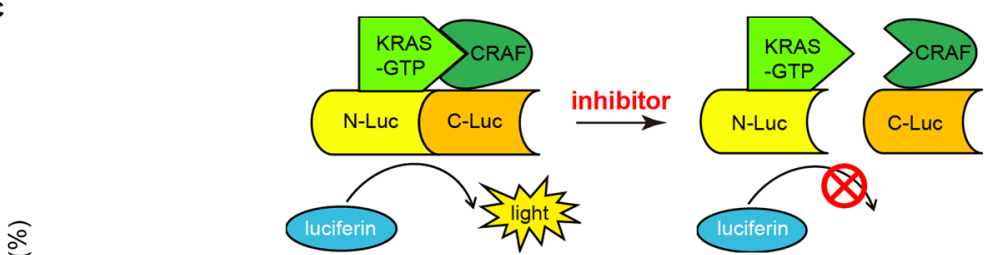

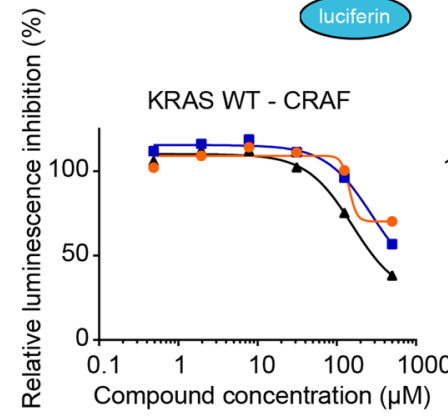

KRAS G12C - CRAF

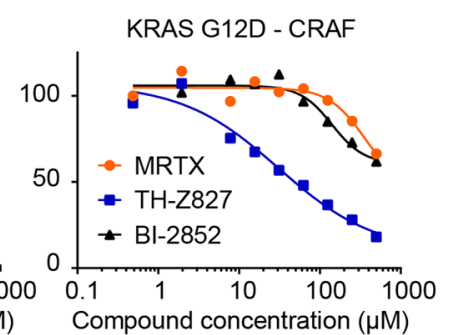

Fig. 4 Mutant-selectivity of the KRAS(G12D) inhibitor TH-Z827. a Binding affinities between TH-Z827 and GDP- or GMPPNP-bound KRAS (WT, G12C, or G12D). b SOS-catalyzed KRAS(G12C) or KRAS(G12D) nucleotide exchange assays in the presence of TH-Z827. c Principle of the split-luciferase reporter assay that detects the KRAS-CRAF interaction in lysates from HEK 293T cells, with or without TH-Z827. Two other compounds (the G12C inhibitor MRTX and the pan-RAS inhibitor BI-2852) were included as controls.

interaction assays motivated us to evaluate the potential anti-cancer effects of TH-Z827. The KRAS G12D mutation is the most prevalent mutation form that drives the most prevalent type of pancreatic cancer ${ }^{34}$. In two pancreatic cancer cell lines bearing the KRAS G12D mutation (PANC-1 and Panc 04.03), TH-Z827 conferred antiproliferative effects with $\mathrm{IC}_{50}$ values of 4.4 and $4.7 \mu \mathrm{M}$, respectively (Fig. 5a). Treatment with $\mathrm{TH}-\mathrm{Z} 827$ also reduced the levels of pERK and PAKT in PANC-1 and Panc 04.03 cells (Fig. 5b), confirming that TH-Z827 prevented the activation of MAPK and PI3K/mTOR signaling. We observed that TH-Z835 reduced the pERK level in PANC-1 cells with an $\mathrm{IC}_{50}$ value less than $2.5 \mu \mathrm{M}$ (Fig. 5c), which was more potent than TH-Z827 (Fig. 5b). We next performed 2D adherent, 3D non-adherent, and colony formation assays and observed anti-proliferative effects of TH-Z835 for two KRAS(G12D)-bearing pancreatic cancer cell lines: PANC-1 and KPC (KrasLSL. G12D/+; p53R172H/+; PdxCretg/+). It was notable that the $\mathrm{IC}_{50}$ values of $\mathrm{TH}-\mathrm{Z} 835$ in the colony formation assay were less than $0.5 \mu \mathrm{M}$ (Supplementary Fig. S6a, b).
Further examination of the inhibitor-treated PANC1 cells revealed increased protein levels of $\mathrm{p} 21$ and $\mathrm{p} 27$, as well as decreased levels of CDK2/4/6 and Cyclin D1, indicating that TH-Z835 induces arrest at the G1 phase of the cell cycle (Fig. 5d). Consistent with these western blotting results, flow cytometry also showed an increased population of PANC-1 cells in G1 phase and a decreased population in S phase as compared with the DMSO control group (Fig. 5e). We also used flow cytometry to evaluate apoptosis induction in TH-Z835-treated PANC-1 and KPC cells and found significantly increased proportions of Annexin V-positive cells (Supplementary Fig. S6c, d). We confirmed this finding by immunoblotting which showed that treatment with TH-Z835 led to increased levels of the apoptosis markers including cleaved PARP, cleaved caspase-3, and cleaved caspase-7 (Fig. 5f).

TH-Z835 is associated with off-target effect, likely due to targeting non-KRAS small GTPases

We next tested the antiproliferative effects of $\mathrm{TH}$ Z835 in other non-G12D mutant cancer cell lines, 


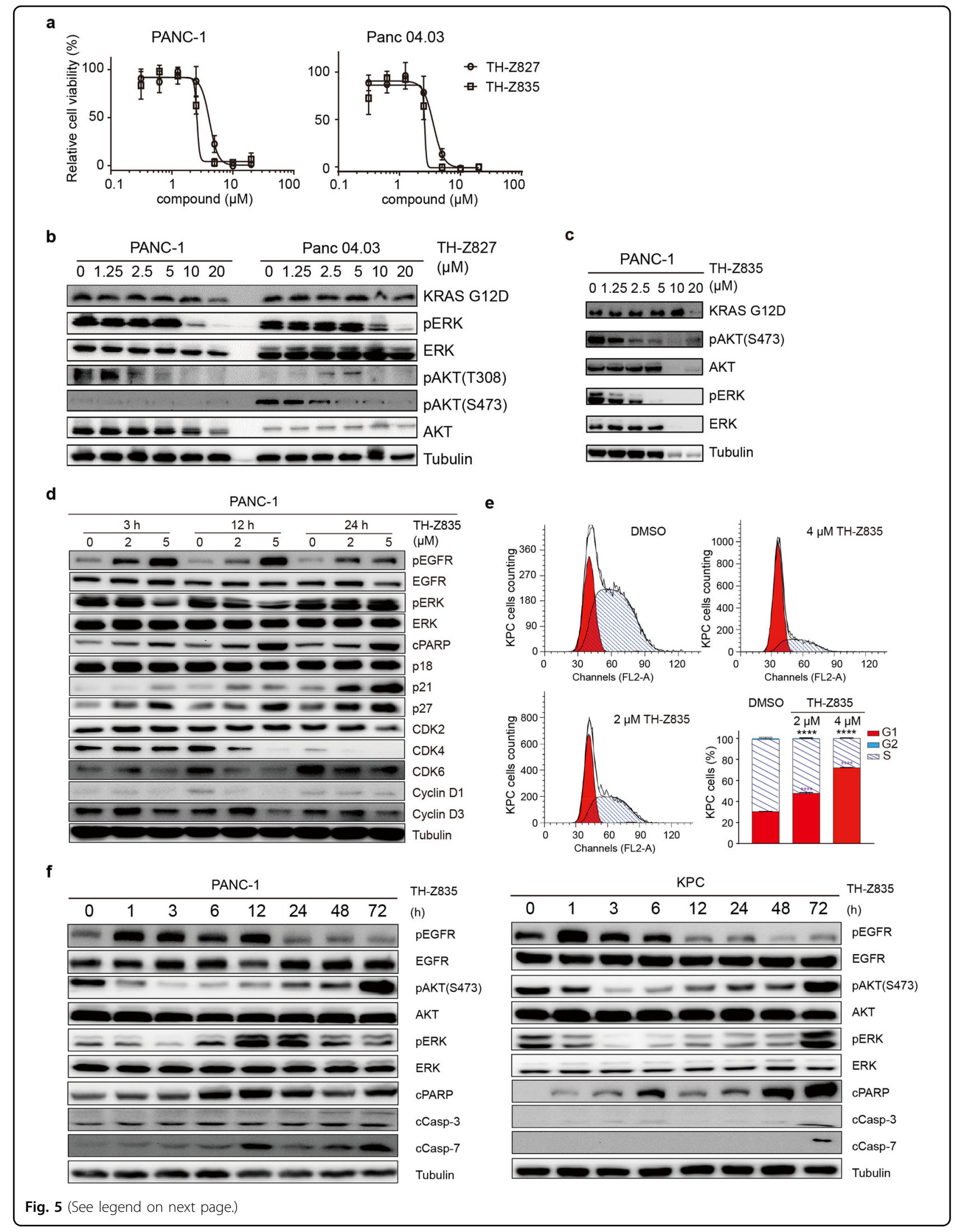


(see figure on previous page)

Fig. 5 Inhibition of KRAS(G12D) mutant cell lines by TH-Z827 and TH-Z835. a Viability assays of PANC-1 and Panc 04.03 cells treated with indicated concentration of TH-Z827 or TH-Z835 for $24 \mathrm{~h}$. b Immunoblotting analysis of ERK and AKT phosphorylation status in PANC-1 (left panel) and Panc 04.03 cells (right panel) treated with TH-Z827 for $3 \mathrm{~h}$. c Immunoblotting analysis of ERK and AKT phosphorylation status in PANC-1 cells treated with TH-Z835 for $3 \mathrm{~h}$. $\mathbf{d}$ Immunoblotting against RTK signaling and cell cycle marker proteins in PANC1 cells after 3-, 12 - or $24 \mathrm{~h}$ treatment with the indicated concentrations of TH-Z835. e Percentages of KPC cells in the G1, S, and G2 phases after treated with TH-Z835 for $24 \mathrm{~h}$ (G2 phase on the top, less than 2\%). Data are shown as means \pm SEM. $n=3$, two-tailed Student's $t$-test, ${ }^{* * * *} P<0.0001$. $\mathbf{f}$ Immunoblotting of EGFR, ERK, AKT phosphorylation, cleaved PARP (CPARP), cleaved Caspase-3 (cCasp-3), and cleaved Caspase-7 (cCasp-7) in PANC-1(left panel) and KPC (right panel) cells treated with TH-Z835 $(5 \mu \mathrm{M})$.
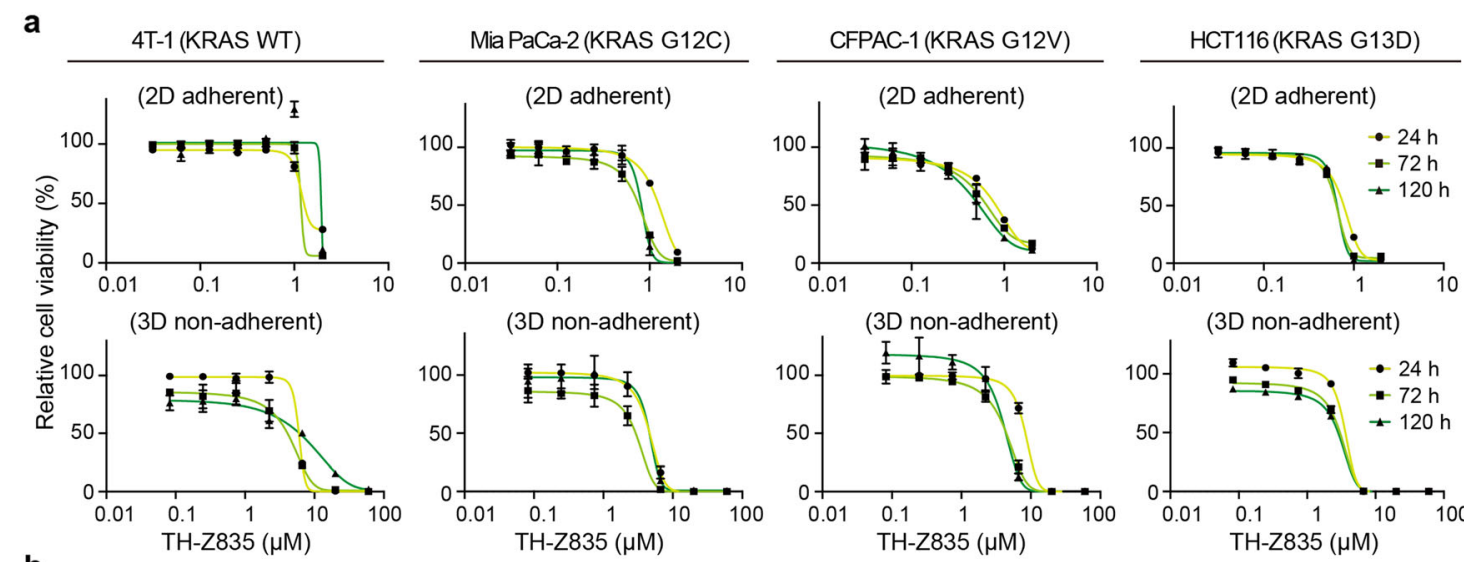

b
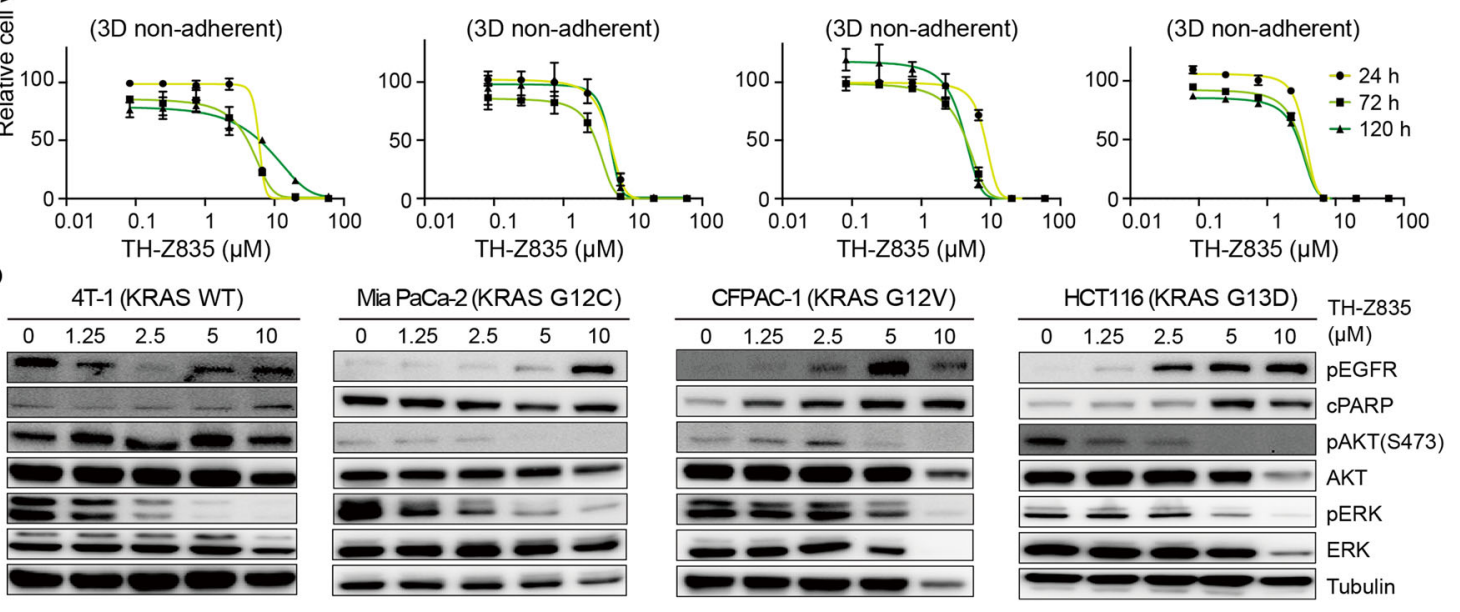

Fig. 6 Anti-proliferative effects and signaling inhibition of TH-Z835 on non-KRAS(G12D) cells. a Cell viability assays of 4T1, MIA PaCa-2, CFPAC1, and HCT116 cells with indicated concentration of TH-Z835 for $24 \mathrm{~h}, 72 \mathrm{~h}$, or $120 \mathrm{~h}$ in $2 \mathrm{D}$ adherent assays (upper panel) and 3D non-adherent assays plates (lower panel). b Immunoblotting of EGFR, ERK, AKT phosphorylation, and CPARP in 4T1, MIA PaCa-2, CFPAC-1, and HCT116 cells treated with TH-Z835 for $3 \mathrm{~h}$.

including 4T1 (KRAS(WT)), MIA PaCa-2 (KRAS (G12C)), CFPAC-1 (KRAS(G12V)), and HCT116 (KRAS(G13D)) cells. Different from our expectations based on data of our in vitro protein assay for the KRAS family proteins, these assays showed that TH-Z835 also conferred anti-proliferative effects (Fig. 6a), reduced the pERK and pAKT levels in these cells (Fig. 6b) and induced apoptosis (Supplementary Fig. S7a-d), suggesting off-target effects. One likely explanation is that TH-Z835 targets some non-KRAS small GTPases, especially given that the switch-II regions of these proteins share structural similarity to those of KRAS proteins. It should also be noted that cell proliferation and MAPK signaling induction in diverse cancers have been shown to be regulated by other Ras superfamily proteins, including members of the Rho, Ran, Arf, and Rab families ${ }^{35}$.

\section{In vivo antitumor activity and combination with PD-1 therapy}

Our in vivo testing of KRAS(G12D) inhibitors was conducted in two xenograft pancreatic tumor models: $\mathrm{BALB} / \mathrm{c}$ nude mice subcutaneously inoculated with Panc 04.03 cells and C57BL/6 mice inoculated with KPC cells. In the nude mice model, TH-Z827 significantly reduced the tumor volumes compared to the vehicle control, and did so in a dose-dependent manner (Fig. 7a). However, an intraperitoneal dosing of $30 \mathrm{mg} / \mathrm{kg} \mathrm{TH}-\mathrm{Z} 827$ resulted in observed weight loss, again suggesting the potential of offtarget effects (Supplementary Fig. S8a). We next tested 
a
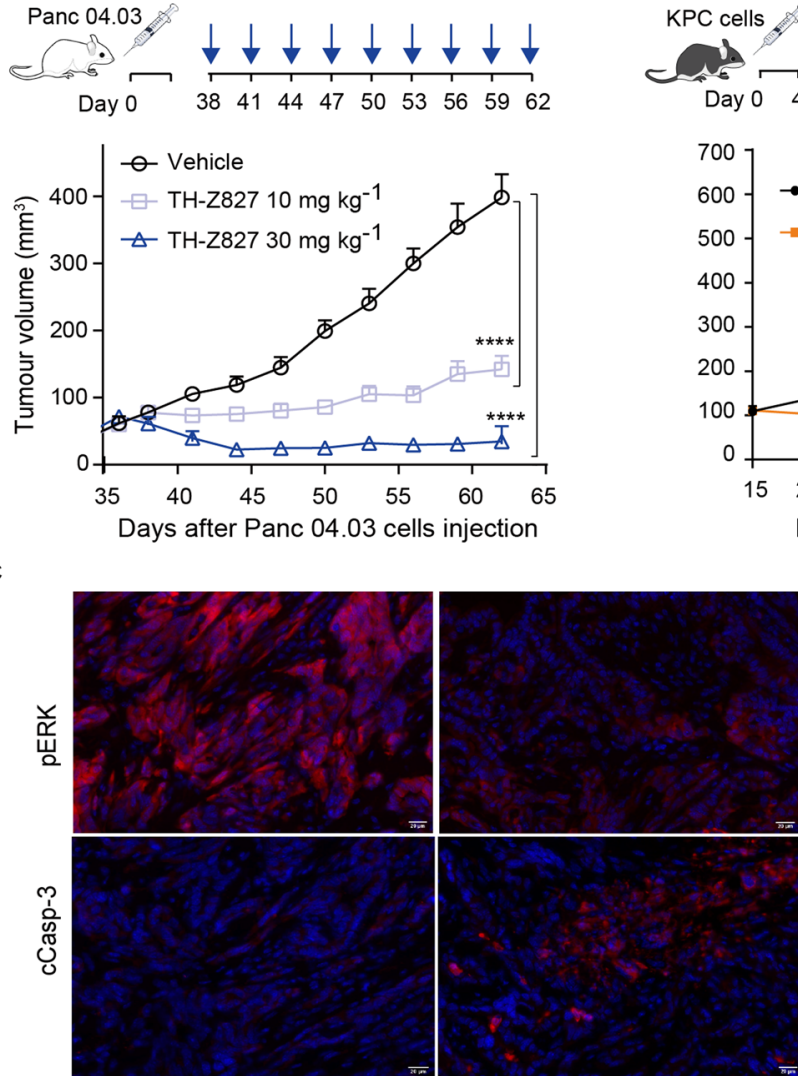

Vehicle
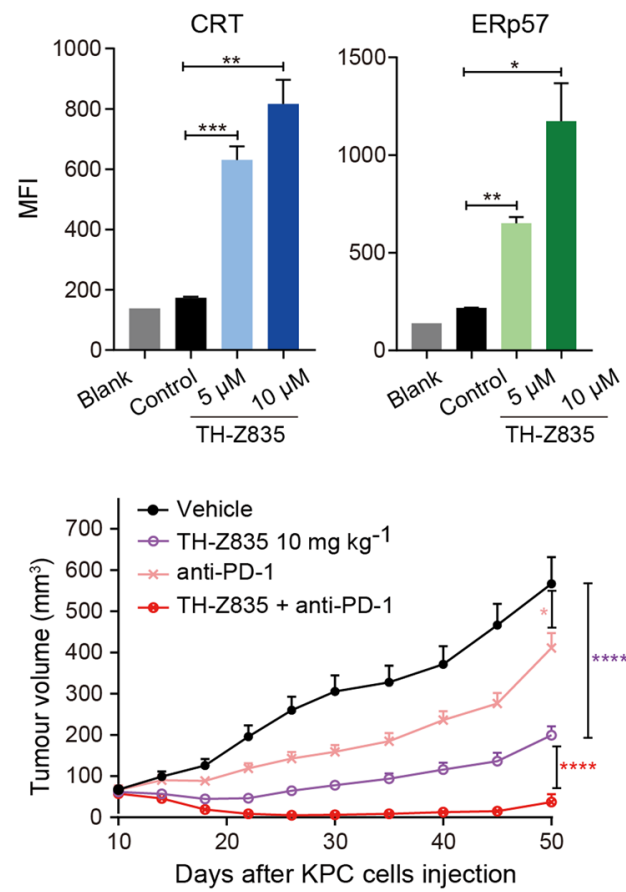

TH-Z835 b
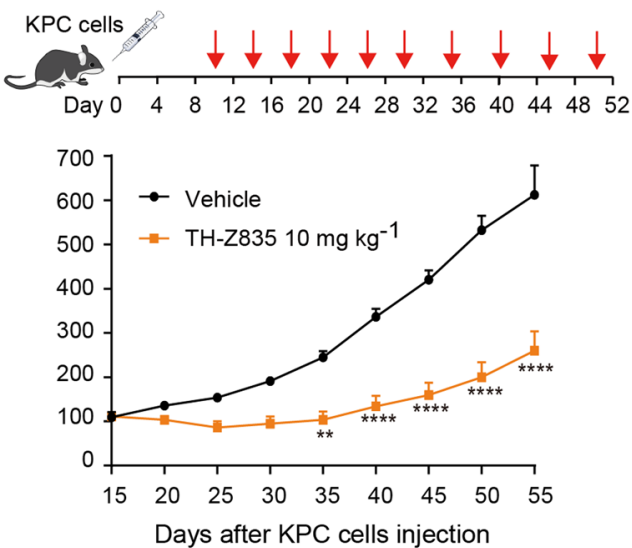
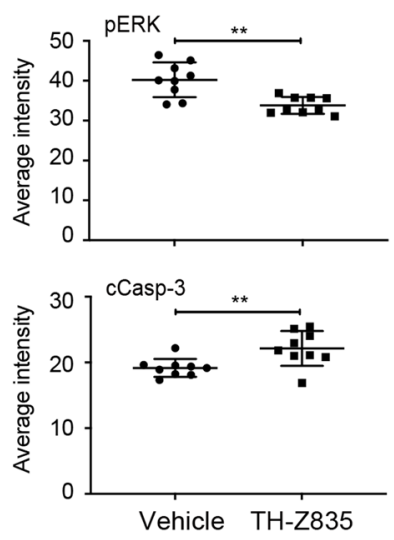
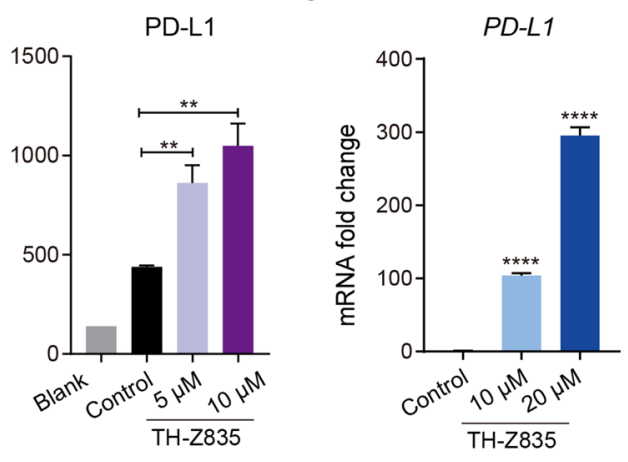

TH-Z835

Fig. 7 (See legend on next page.) 
(see figure on previous page)

Fig. 7 Anti-tumor effects of the KRAS(G12D) inhibitors alone and in combination with anti-PD-L1 antibody. a Nude mice injected with Panc 04.03 cells at Day 0 were later intraperitoneally injected with TH-Z827 (at $10 \mathrm{mg} / \mathrm{kg}$ or $30 \mathrm{mg} / \mathrm{kg}$ ) according to the indicated dosage schedule (blue arrow). The tumor volumes (means \pm SEM,$n=10$ ) were measured with digital calipers and assessed using one-way ANOVA followed by Dunnett's test, ${ }^{* * *} P_{\text {adj }}<0.0001$. b Mice were injected with KPC cells at Day 0 and TH-Z835 at Day 10. The tumor volumes (means \pm SEM, $n=10$ ) were assessed using one-way ANOVA followed by Dunnett's test, ${ }^{* *} P_{\text {adj }}<0.01,{ }^{* * * *} P_{\text {adj }}<0.0001$. c Left panel: Immunofluorescence (IF) analysis of pERK and cleaved Caspase-3 in tumor sections from C57BL/6 mice (b) treated with TH-Z835 or vehicle. Scale bar, $20 \mu \mathrm{m}$. Right panel: quantification of IF-positive staining. Data are shown as means \pm SEM. $n=9$, two-tailed Student's $t$-test, ${ }^{* *} P<0.01$. d Flow cytometry analysis of the immunogenic cell death (ICD) markers CRT and ERp57 and the immune checkpoint protein PD-L1 on the surface of KPC cells after $24 \mathrm{~h}$ treatment with TH-Z835. Data are shown as means \pm SEM $(n=3)$, two-tailed Student's $t$-test, ${ }^{*} P<0.05$, ${ }^{* *} P<0.01$, ${ }^{* * *} P<0.001$. e mRNA expression level of $P D D-L 1$ in PANC-1 cells after $24 \mathrm{~h}$ treatment with TH-Z835. Data are shown as means \pm SEM $(n=3)$, two-tailed Student's $t$-test, ${ }^{* * * *} P<0.0001$. f C57BL/6 mice were injected with KPC cells at Day 0, after which TH-Z835, anti-PD-1 antibody, or a combination therapy (10 mg/kg TH-Z835 and $100 \mu \mathrm{mg}$ per dose anti-PD-1 antibody) was intraperitoneally administered. The tumor volumes (means $\pm \mathrm{SEM}, n=5$ ) were assessed using one-way ANOVA followed by Dunnett's test, * $P_{\text {adj }}<0.05,{ }^{* * *} P_{\text {adj }}<0.0001$.

the antitumor activity of $\mathrm{TH}-\mathrm{Z} 835$ in the $\mathrm{C} 57 \mathrm{BL} / 6$ mice model. The tumor volume was also reduced compared with the vehicle group (Fig. 7b). In addition, immunohistochemical analysis of tumor sections revealed an increased expression of cleaved caspase- 3 and a decreased expression of pERK1/2 (Fig. 7c; Supplementary Fig. S8b), indicating that $\mathrm{TH}-\mathrm{Z} 835$ induces apoptosis and inhibits MAPK signaling in vivo.

Recent studies showed that besides its function in cancer cells, KRAS oncogenic signaling can orchestrate the immune status of the tumor microenvironment ${ }^{36-38}$. Specifically, studies using murine pancreatic cancer models showed that KRAS can drive immune evasion

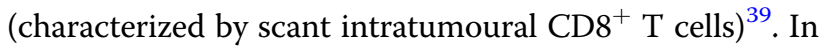
addition, activated MAPK signaling may also be involved in the immunosuppressive tumor microenvironment ${ }^{40}$. We first tested the effect of TH-Z835 treatment on KPC cells and found increased $P D-L 1$ mRNA expression and increased levels of the immunogenic cell death markers CRT and ERp57 on the cell surface (Fig. 7d, e; Supplementary Fig. S8c). We next tested the efficacy of a combination therapy comprising TH-Z835 and an anti-PD-1 antibody for C57BL/6 mice inoculated with KPC cells. Indeed, a combination treatment led to a statistically significant decrease in tumor volume as compared to either of the mono-therapies (Fig. 7f). This synergism could also be observed in a combination therapy comprising TH-Z827 and the anti-PD-1 antibody (Fig. S8d).

\section{Discussion}

Aiming to develop inhibitors for KRAS(G12D), a more common KRAS mutant variant, we synthesized a series of small molecules that can form a salt bridge with Asp12 residue, mimicking the acryloyl-cysteine interaction of KRAS(G12C) and its inhibitors, and characterized their activities both in vitro and in vivo. Unlike covalent KRAS (G12C) inhibitors that selectively bind to GDP-bound proteins, both our computational and ITC investigations suggest that these inhibitors that can form salt bridge bind to both GDP-bound and GTP-bound KRAS(G12D). We also solved crystal structures of KRAS(G12D) with series of potent inhibitors (TH-Z816, TH-Z827, and TH-Z835). Our structural data confirmed that these inhibitors can induce the formation of an allosteric pocket under the KRAS(G12D) switch-II region, similar to reported findings for the covalent KRAS(G12C) inhibitors ${ }^{8,9,13,15}$. In addition, the crystal structures revealed that these inhibitors are able to bind to the GMPPNP-bound KRAS (G12D). That is, whereas steric clashing renders the G12C inhibitors incapable of targeting GTP-bound KRAS $(\mathrm{G} 12 \mathrm{C})^{9}$, our salt-bridge forming inhibitors have sufficient space to target Asp12 for both GDP-bound and GTPbound KRAS(G12D). Notably, the discovery of G12C inhibitors might result in a stereotype that targeting the GDP-bound (inactive) KRAS may be a more viable approach than targeting the GTP-bound (active) KRAS ${ }^{5}$. Now, using the inhibitors developed in the present study, it should be possible to launch hypothesis-driven basic and translational investigations about the differential impacts and therapeutic consequences of targeting active KRAS mutant.

We detected no binding or inhibitory effects of our compounds towards the KRAS(WT) or KRAS(G12C) proteins, whereas our data show that these KRAS(G12D) inhibitors can efficiently disrupt the KRAS-CRAF interaction. These molecules also disrupt the activation of MAPK and PI3K/mTOR signaling in diverse cancer cells and display anti-proliferative and anti-tumor effects. However, despite this apparently clear picture from our in vitro work, we stress that this efficacy is not fully dependent on KRAS mutation status: our data from assays with diverse cancer cells bearing WT KRAS revealed a more complex interaction pattern. Our experiments with xenograft model of pancreatic tumors showcase the very promising efficacy and synergistic potential for combination therapy of TH-Z835; nevertheless, our observation of weight loss again suggested off-target impacts. A very likely explanation is that the inhibitors bind to and inhibit 
Rho, Ran, Arf, and/or Rab subfamily proteins that also function in regulating cancer cell proliferation and MAPK signaling. In sum, our study has demonstrated proof-ofconcept for strategy based on the formation of a saltbridge and induced-fit pocket to achieve KRAS(G12D) inhibition, which warrants future medicinal chemistry efforts to obtain better specificity and optimal efficacy.

\section{Methods}

\section{Protein expression and purification}

The gene encoding KRAS(G12D) (residues 1-169) was chemically synthesized and cloned into pET28a vector with NdeI and XhoI restriction sites. A construct for the recombinant KRAS(G12D) was transformed into E. coli BL21 (DE3). After the bacterial growth to an $\mathrm{OD}_{600}$ of 0.6 , induction was carried out using $1 \mathrm{mM}$ isopropyl- $\beta$-D-thiogalactopyranoside (IPTG) $\left(16^{\circ} \mathrm{C}\right.$ for $\left.24 \mathrm{~h}\right)$. The cells were lysed and recombinant KRAS(G12D) was purified using a HisTrap HP (GE Healthcare, 29-0510-21) column. The hexahistidine tag was then removed by TEV-protease and crude protein was further purified. The mature protein was concentrated to $3.5 \mathrm{mg} / \mathrm{mL}$, and the molecular mass of protein was determined by SDS-PAGE $(\sim 21 \mathrm{kDa})$. The KRAS protein sequence is 'MTEYKLVVVG ADGVGK SALT IQLIQNHFVD EYDPTIEDSY RKQVVIDGET CLL DILDTAG QEEYSAMRDQ YMRTGEGFLC VFAINNT KSF EDIHHYREQI KRVKDSEDVP MVLVGNKCDL PSRTVDTKQA QDLARSYGIP FIETSAKTRQ GVDDAF YTLV REIRKHKEK'. SOS, KRAS(WT), and KRAS(G12C) were expressed and purified in a similar way as KRAS (G12D). The plasmid of SOS was provided by Professor Niu Huang of National Institute of Building Sciences.

\section{X-ray crystallography}

The protein was further concentrated to $40 \mathrm{mg} / \mathrm{mL}$ for the X-ray crystallography study. For TH-Z816, KRAS(G12D) protein was directly used for the following procedure. For TH-Z827 and TH-Z835, the endogenous GDP of KRAS(G12D) was exchanged with GMPPNP catalyzed by EDTA. Next, using the vapordiffusion method, thin plates were observed after a week at $20^{\circ} \mathrm{C}$ under the crystallization conditions of $0.2 \mathrm{M}$ sodium acetate, $0.1 \mathrm{M}$ Tris, $\mathrm{pH} 8.5,26 \%(\mathrm{w} / \mathrm{v})$ PEG 3350. To obtain the complex structures, the protein crystals were soaked into $2 \mathrm{mM}$ inhibitor for $6 \mathrm{~h}$. The soaked crystals were cryoprotected in the mother liquor supplemented with $10 \%$ glycerol prior to flashfreezing. The X-ray diffraction data were obtained at the in-house beamline BRUKER D8 VENTURE at Hubei University. Datasets were initially processed with PROTEUM3 v2020.6, solved by molecular replacement using Phaser with KRAS (PDB ID: 4EPR), and refined to the indicated statistics using PHENIX 1.10.1-2155 and Coot $0.8 .3^{41}$. The figures were drawn using PyMOL.

\section{EDTA-catalyzed nucleotide exchange}

Endogenous nucleotides in KRAS were exchanged with GDP (Sigma, G7127), GMPPNP (Sigma, G0635), mantGDP (Jena Bioscience, NU-204S), or mantGMPPNP (Jena Bioscience. NU-207S) using a previously reported EDTA-catalyzed procedure ${ }^{42,43}$. Briefly, KRAS(G12D) protein $(10 \mu \mathrm{M})$ was incubated with incoming nucleotide $(200 \mu \mathrm{M})$ and EDTA $(2.5 \mathrm{M})$ for $1.5 \mathrm{~h}$ at room temperature. After incubation, the sample was put on ice for $2 \mathrm{~min}$, and then $\mathrm{MgCl}_{2}$ (5 $\mathrm{mM}$ final) was added to stop the reaction. Excess unbound nucleotide was removed using a NAP-5 column (GE Healthcare, 17085302).

\section{SOS-catalyzed nucleotide exchange}

For studies with mant-nucleotide loaded protein, $12 \mu \mathrm{L}$ of the protein $(1.25 \mu \mathrm{mol} / \mathrm{L})$ in reaction buffer containing $1 \mathrm{mmol} / \mathrm{L}$ of a given incoming nucleotide (GDP or GMPPNP) was added to a 96-well half-area microplate (Corning 3686). After incubation with compounds for $10 \mathrm{~min}$, reactions were initiated by the addition of $3 \mu \mathrm{L}$ of SOS $(10 \mu \mathrm{mol} / \mathrm{L})$; fluorescence $\left(\lambda_{\mathrm{ex}}=355 \mathrm{~nm}, \lambda_{\mathrm{em}}=\right.$ $460 \mathrm{~nm}$ ) was monitored for $45 \mathrm{~min}$ at $60 \mathrm{~s}$ intervals with a multimode microplate reader (PerkinElmer, EnVision). For the exchange assays with incoming mant-nucleotide, $12 \mu \mathrm{L}$ of the purified protein $(1.25 \mu \mathrm{mol} / \mathrm{L})$ was used (in reaction buffer containing $1 \mu \mathrm{mol} / \mathrm{L}$ of the indicated incoming nucleotide). Fluorescence data were fitted to a one phase decay model with GraphPad Prism 7.0.

\section{Molecular docking}

Compounds were constructed using the Schrödinger Maestro 3D sketcher, and candidate conformations were prepared and generated using LigPrep. Protein structures were optimized using Protein Preparation Wizard software, with docking grids profiles generated around the ligand. Docking was performed using Glide software, with standard precision. The force field was OPLS_2005.

\section{ITC assays}

ITC experiments were carried out at $25^{\circ} \mathrm{C}$ with 19 injections, $2 \mu \mathrm{L}$ per injection, and $150 \mathrm{~s}$ intervals using a MicroCal PEAQ-ITC instrument (GE Healthcare). Buffer was exchanged into $25 \mathrm{mM}$ Tris- $\mathrm{HCl}, 100 \mathrm{mM} \mathrm{NaCl}$, $5 \mathrm{mM} \mathrm{MgCl}_{2}, 1 \mathrm{mM}$ TCEP (Sigma, C4706), 0.05\% Tween20 (Sigma, P1379) and 2.5\% (v/v) DMSO. The protein was loaded into a cell, and the compound was titrated. Reference power was set to $10 \mu \mathrm{cal} / \mathrm{s}$, and the compound solution was titrated at $150 \mathrm{~s}$. Data were fitted into a one site model, and $\mathrm{KD}, \mathrm{N}, \Delta G, \Delta H$, and $-T \Delta S$ values were calculated using MicroCal Analysis software.

\section{KRAS-CRAF interaction assay}

Three HEK 293T cell lines expressing CRAF together with KRAS(WT), KRAS(G12C), or KRAS(G12D) were 
generated. Cells were cultured on six-well plates $(2.5 \times$ $10^{5}$ per well) and treated with $1 \mu \mathrm{g} / \mathrm{mL}$ doxycycline for $24 \mathrm{~h}$. Then each well was treated with $200 \mu \mathrm{L}$ lysis buffer (Promega, E2661) and centrifuged at $4{ }^{\circ} \mathrm{C}$ for $5 \mathrm{~min}$. The supernatant was collected and incubated with compounds (TH-Z827, MRTX, or BI-2852) for $10 \mathrm{~min}$. Then $20 \mu \mathrm{L}$ luciferin substrate buffer (Promega, E2510) was added and the samples were incubated for $10 \mathrm{~min}$ before luminescence was measured with a multimode microplate reader (PerkinElmer, EnVision). Data were fitted into an inhibitor-response model to get $\mathrm{IC}_{50}$ values.

\section{D cell viability assays}

All the cell lines used in this study were obtained from ATCC. PANC-1 and Panc 04.03 cells were seeded onto 96-well microplates $\left(5 \times 10^{3}\right.$ cells per well) and cultured for 24 $\mathrm{h}$ with DMEM medium (Gibco, 11960-051) supplemented with 10\% FBS (Biological Industries, 04-001-1) and $1 \%$ Pen/Strep (Beyotime, C0222). After treatment with compounds (TH-Z827, MRTX, or BI-2852), cells were incubated for another $24 \mathrm{~h}$. Cell Counting Kit-8 reagents (Beyotime, C0042) were added and the samples were incubated for another $1 \mathrm{~h}$. Cell viability was measured at OD $(450 \mathrm{~nm})$ using a multimode microplate reader (PerkinElmer, EnVision). Data were fitted into an inhibitor-response model to get $\mathrm{IC}_{50}$ values.

\section{D cell viability assays}

For comparison of anti-growth activity, a CellTiter-Glo (CTG) 3D cell viability assay (Promega, G9682) was used. Cells $\left(1 \times 10^{4}\right.$ cells per well) were seeded (using the same media) in ultra-low attachment surface 96-well format plates (Corning Costar \#3474). On the day after plating, cells were treated with a 7 point three-fold dilution series of indicated compounds $(200 \mu \mathrm{L}$ final volume per well), and cell viability was monitored at 1, 3, 5 days according to the manufacturer's recommended instructions, after which $50 \mu \mathrm{L}$ of CellTiter-Glo reagent was added. Samples were then vigorously mixed, covered, and placed on a plate shaker for $20 \mathrm{~min}$ to ensure complete cell lysis prior to assessment of luminescent signal.

\section{Colony formation assay}

KPC or PANC1 $\left(1 \times 10^{3}\right.$ cells per well $)$ were seeded in the six-well plates in triplicate. After overnight incubation, cells were treated with various concentrations of TH-Z835 or vehicle (DMSO), and allowed to grow for 10 to 14 days, during which the medium was changed every 3 days. After $10-14$ days, cells were fixed by $4 \%$ paraformaldehyde (Leagene, DF0135), and cell colonies were stained by crystal violet solution (Beyotime, C0121) for $30 \mathrm{~min}$. After washing with water, $10 \%$ methanol-acetic acid solution was added to dissolve the stained cell precipitation, and the absorbance was measured at $590 \mathrm{~nm}$.

\section{Cell cycle detection by flow cytometry}

Cells were seeded in six-well plate and synchronized with serum-free medium for $24 \mathrm{~h}$. Next, the cells were released in a complete medium containing either DMSO or TH-Z835, and collected for analysis at $24 \mathrm{~h}$. For cell cycle analysis, the cell DNA was stained with propidium iodide (PI) using cell cycle and apoptosis analysis kit (Beyotime, C1052). Briefly, cells were harvested by trypsinization and fixed with cold $75 \%$ ethanol at $4{ }^{\circ} \mathrm{C}$ overnight. The fixed cells were collected and suspended in PBS containing $10 \mu \mathrm{g} / \mathrm{mL}$ PI and $10 \mu \mathrm{g} / \mathrm{mL}$ RNase A, and then incubated at room temperature for $30 \mathrm{~min}$. DNA content was analyzed by the BD FACS Calibur (BD Biosciences), and each histogram was constructed with the data from 10,000 to 20,000 events. The data were analyzed and presented as percentages of total gated cells using the Modfit $\mathrm{LT}^{\mathrm{TM}}$ Software (BD Biosciences).

\section{Western blotting}

PANC-1 and Panc 04.03 cells were cultured on six-well plates $\left(5 \times 10^{5}\right.$ cells per well) and treated with TH-Z827 for $3 \mathrm{~h}$. Protein samples were prepared, electrophoresed (10\% SDS-PAGE), and transferred to a polyvinylidene fluoride membrane. Individual proteins were detected using antiRAS G12D (Cell Signaling Technology, 14429), anti-pERK1/ 2 (Cell Signaling Technology, 4370), anti-ERK1/2 (Cell Signaling Technology, 4695), anti-pAKT(Thr308) (Cell Signaling Technology, 2965), anti-pAKT(Ser473) (Cell Signaling Technology, 4060), anti-AKT (Cell Signaling Technology, 2920), anti-tubulin (Proteintech, 66240-1-Ig), anti-EGF Receptor (Cell Signaling Technology, 4267), anti-phosphoEGF Receptor (Tyr1068) (Cell Signaling Technology, 3777), anti-cleaved PARP (Asp214) (Cell Signaling Technology, 9541), anti-cleaved Caspase-3 (Asp175) (Cell Signaling Technology, 9661), anti-cleaved Caspase-7 (Asp198) (Cell Signaling Technology, 9491) antibodies and cell cycle regulation antibody sampler kit (Cell Signaling Technology, 9932). For detection of MAPK signaling in KRAS(G12D) or non-G12D mutant cells, cells were cultured on six-well plates $\left(5 \times 10^{5}\right.$ cells per well $)$ and treated with a serial titration of TH-Z835 or TH-Z827 for $3 \mathrm{~h}$. For apoptosis, RTK feedback regulation, or cell-cycle arrest assay, PANC-1 or KPC were cultured on six-well plates $\left(5 \times 10^{5}\right.$ cells per well) and treated with a serial titration of TH-Z835 at 0, 1, 3, $6,12,24,48$, and $72 \mathrm{~h}$ (for apoptosis) or at 3,12 , and $24 \mathrm{~h}$ (for RTK feedback regulation and cell-cycle arrest assay).

\section{Apoptosis detection by Annexin V binding}

Cells $\left(5 \times 10^{5}\right.$ cells per well) were seeded in six-well plate overnight. Next, the cells were treated with either DMSO or TH-Z835 for $12 \mathrm{~h}$ or $24 \mathrm{~h}$. For apoptosis analysis, cells were harvested by trypsinization and washed twice with ice-cold PBS, then stained with Annexin V-FITC and PI by Apoptosis Detection Kit (Beyotime, 
C1062) in the dark at room temperature for $10 \mathrm{~min}$. Then cells were analyzed with the BD FACS Ariall and FlowJo software.

\section{Apoptosis detected by WB}

For apoptosis, RTK feedback regulation, or cell-cycle arrest assay, PANC-1 or KPC were cultured on six-well plates $\left(5 \times 10^{5}\right.$ cells per well $)$ and treated with a serial titration of TH-Z835 at various time points for up to $72 \mathrm{~h}$.

\section{Immunogenic cell death and PD-L1 detection by flow cytometry}

The cells were treated with TH-Z835 as indicated for $24 \mathrm{~h}$ before harvesting. After washing twice in cold PBS, cells were incubated for $30 \mathrm{~min}$ with anti-PD-L1 (1:1000, ab213480, Abcam), anti-CRT (1:1000, ab2907, Abcam), or anti-ERp57 (1:1000, ab10287, Abcam) antibody, diluted in cold blocking buffer (2\% FBS in PBS), followed by washing and incubation with the Alexa Fluor 488-labeled secondary antibody (1:1000, ZF-0511, ZSGB-BIO) for $30 \mathrm{~min}$. Then cells were analyzed with the BD FACS AriaII and FlowJo software.

\section{Real-Time quantitative PCR}

PANC-1 was treated with multiple doses of TH-Z835 for $24 \mathrm{~h}$. Total RNA was extracted with TRIzol reagent (CWBIO, CW0580). cDNA was prepared using $1 \mu \mathrm{g}$ of RNA with a cDNA Synthesis Kit (Yeasen, 11141ES10). SYBR-green-based qPCR was performed using primers for PD-L1 (forward, TTTGCTGAACGCCCCATACA; reverse, TTGGTGGTGGTGGTCTTACC), GAPDH (forward, GAGTCAACGGATTTGGTCGT; reverse, TT GATTTTGGAGGGATCTCG). Gene expression was calculated by the comparative $\Delta \Delta C T$ method with the GAPDH for normalization.

\section{Mouse models}

BALB/c nude mice were subcutaneously injected with Panc 04.03 cells $\left(1 \times 10^{7}\right.$ per dose $)$ at Day 0 . Mice were randomized when the mean tumor volume was $\sim 70 \mathrm{~mm}^{3}$. Each group of mice $(n=10)$ was intraperitoneally injected with $\mathrm{PBS}, 10 \mathrm{mg} / \mathrm{kg}$ or $30 \mathrm{mg} / \mathrm{kg} \mathrm{TH}-\mathrm{Z} 827$ according to the dosage schedule from Day 38 to Day 62.

C57BL/6 mice were subcutaneously injected with KPC (KrasLSL.G12D/ + ; p53R172H/+; PdxCretg/+) cells $\left(5 \times 10^{5}\right.$ per dose $)$. Mice were randomized when the mean tumor volume was $\sim 20 \mathrm{~mm}^{3}$. Mice of each group $(n=10)$ were intraperitoneally injected with PBS, antiPD-1 antibody (100 $\mu$ g per dose, Bio X Cell, BE0033-2), $10 \mathrm{mg} / \mathrm{kg} \mathrm{TH}-\mathrm{Z} 827$, or a combination $(10 \mathrm{mg} / \mathrm{kg} \mathrm{TH}-$ Z827 and anti-PD-1 antibody) according to a pre-defined dosage schedule from Day 7 to Day 38. Polyclonal Armenian hamster IgG (Bio X Cell, BE0091) was used as a control antibody.
Statistical analysis of differences in mean tumor volume between vehicle and treated groups were assessed using one-way ANOVA test conducted in GraphPad Prism. P value $<0.05$ was considered statistically significant.

\section{Immunohistochemistry and immunofluorescence}

Tumor samples were obtained after treatment for 30 days, and fixed in $4.0 \%$ paraformaldehyde solution (Leagene, DF0135), embedded in paraffin, and cut into $4 \mu \mathrm{m}$ sections. Sections were used for Immunohistochemistry (IHC) and Immunohistochemistry (IF) according to the standard procedures. The IHC staining protocol was briefly described as follows: the slides were routinely deparaffinized, rehydrated, subjected to antigen retrieval, and incubated in 3\% hydrogen peroxide to block endogenous peroxidase. Subsequently, the slides were blocked with $3 \%$ BSA and incubated with primary antibodies against pERK1/2 (1:500, Cell Signaling Technology, 4370) or cleaved Caspase-3 (1:400, Cell Signaling Technology, 9661) at $4{ }^{\circ} \mathrm{C}$ overnight, and with polymerHRP-conjugated anti-rabbit secondary antibody (1:200S, Servicebio, GB23303). Then, the sections were stained with DAB kit (Servicebio, G1211), counterstained with hematoxylin, dehydrated, and cover-slipped. For IF staining, slides were incubated with primary antibodies against pERK1/2 (1:200, Cell Signaling Technology, 4370), cleaved Caspase-3 (1:200, Cell Signaling Technology, 9661) at $4{ }^{\circ} \mathrm{C}$ overnight, and with CY3-conjugated antirabbit secondary antibody (1:300, Servicebio, GB21303). Then, the sections were counterstained with antifading agent DAPI (Beyotime, P0131).

\section{Acknowledgements \\ We acknowledge Professor Niu Huang for providing the plasmid of SOS and Dr. Yifeng Xia for donating the HEK 293T cell lines used for detecting KRAS-CRAF interactions. This work was supported by the National Key R\&D Program of China (2021YFC2100300), the Beijing Natural Science Foundation (Z190015), the National Natural Science Foundation of China (81991492), the Beijing Advanced Innovation Center for Structural Biology, the Beijing Advanced Innovation Center for Human Brain Protection and Tsinghua University Spring Breeze Fund.}

\section{Author details \\ ${ }^{1}$ School of Pharmaceutical Science, Tsinghua-Peking Center for Life Sciences, Tsinghua University; Beijing Advanced Innovation Center for Human Brain Protection, Beijing, China. ${ }^{2}$ Joint Graduate Program of Peking-Tsinghua-NIBS, School of Life Sciences, Tsinghua University, Beijing, China. ${ }^{3}$ State Key Laboratory of Bio-catalysis and Enzyme Engineering, Hubei Collaborative Innovation Center for Green Transformation of Bio-Resources, School of Life Sciences, Hubei University, Wuhan, China. ${ }^{4}$ Department of Biomedical Engineering, School of Medicine, Tsinghua-Peking Center for Life Science, Tsinghua University, Beijing, China}

\section{Author contributions}

Y.Z. initiated, designed, and supervised the project, and wrote the manuscript. Z.M. designed, synthesized compounds, performed enzymatic assays, and wrote the manuscript. H.X., Z.M., Y.Z. and Y.D. performed the cellular experiments. H.X. performed the animal experiments and wrote the manuscript. P.S., Yu Y., J.X., L.Z., and Yunyun Y. performed protein purification and structural studies. Y.S., X.L., C.-C.C., and R.-T.G. contributed to the study design and discussion. 


\section{Data availability}

Most of the data generated or analyzed during this study are included in this article or available as supplementary information. X-ray crystallographic coordinates and structure factor files have been deposited in the Protein Data Bank (PDB ID: 7EW9, 7EWA, 7EWB). Other data that support the findings of this study are available from the corresponding authors upon request.

\section{Conflict of interest}

The authors declare no competing interest.

\section{Publisher's note}

Springer Nature remains neutral with regard to jurisdictional claims in published maps and institutional affiliations.

Supplementary information The online version contains supplementary material available at https://doi.org/10.1038/s41421-021-00368-w.

Received: 22 June 2021 Accepted: 23 December 2021

Published online: 25 January 2022

\section{References}

1. Cox, A. D., Fesik, S. W., Kimmelman, A. C., Luo, J. \& Der, C. J. Drugging the undruggable RAS: Mission possible? Nat. Rev. Drug Discov. 13, 828-851 (2014).

2. Waters, A. M. \& Der, C. J. KRAS: The critical driver and therapeutic target for pancreatic cancer. Cold Spring Harb. Perspect. Med. 8, a031435 (2018).

3. Cherfils, J. \& Zeghouf, M. Regulation of small GTPases by GEFs, GAPs, and GDls. Physiol. Rev. 93, 269-309 (2013).

4. McCormick, F. Targeting KRAS directly. Annu. Rev. Cancer Biol. 2, 81-90 (2018).

5. Ostrem, J. M. \& Shokat, K. M. Direct small-molecule inhibitors of KRAS: From structural insights to mechanism-based design. Nat. Rev. Drug Discov. 15, 771-785 (2016).

6. Hall, B. E., Bar-Sagi, D. \& Nassar, N. The structural basis for the transition from Ras-GTP to Ras-GDP. Proc. Natl. Acad. Sci. USA 99, 12138-12142 (2002).

7. Hunter, J. C. et al. Biochemical and structural analysis of common cancerassociated KRAS mutations. Mol. Cancer Res. 13, 1325-1335 (2015).

8. Ostrem, J. M., Peters, U., Sos, M. L., Wells, J. A. \& Shokat, K. M. K-Ras(G12C) inhibitors allosterically control GTP affinity and effector interactions. Nature 503, 548-551 (2013).

9. Patricelli, M. P. et al. Selective inhibition of oncogenic KRAS output with small molecules targeting the inactive state. Cancer Discov. 6, 316-329 (2016).

10. Janes, M. R. et al. Targeting KRAS mutant cancers with a covalent G12Cspecific inhibitor. Cell 172, 578-589 (2018).

11. Canon, J. et al. The clinical KRAS(G12C) inhibitor AMG 510 drives anti-tumour immunity. Nature 575, 217-223 (2019).

12. Hallin, J. et al. The KRAS(G12C) inhibitor MRTX849 provides insight toward therapeutic susceptibility of KRAS-mutant cancers in mouse models and patients. Cancer Discov. 10, 54-71 (2020).

13. Lito, P., Solomon, M., Li, L. S., Hansen, R. \& Rosen, N. Allele-specific inhibitors inactivate mutant KRAS G12C by a trapping mechanism. Science $351,604-608$ (2016).

14. Kettle, J. G. et al. Structure-based design and pharmacokinetic optimization of covalent allosteric inhibitors of the mutant GTPase KRAS(G12C). J. Med. Chem. 63, 4468-4483 (2020).

15. Fell, J. B. et al. Identification of the clinical development candidate MRTX849, a covalent KRAS(G12C) inhibitor for the treatment of cancer. J. Med. Chem. 63, 6679-6693 (2020).

16. Hansen, R. et al. The reactivity-driven biochemical mechanism of covalent KRAS(G12C) inhibitors. Nat. Struct. Mol. Biol. 25, 454-462 (2018).

17. Prior, I. A., Lewis, P. D. \& Mattos, C. A comprehensive survey of Ras mutations in cancer. Cancer Res. 72, 2457-2467 (2012).
18. Kessler, D. et al. Drugging an undruggable pocket on KRAS. Proc. Natl. Acad. Sci. USA 116, 15823-15829 (2019).

19. Feng, H. et al. K-Ras(G12D) has a potential allosteric small molecule binding site. Biochemistry 58, 2542-2554 (2019).

20. Welsch, M. E. et al. Multivalent small-molecule pan-RAS Inhibitors. Cell 168, 878-889 (2017).

21. Zhang, Z. et al. GTP-state-selective cyclic peptide ligands of K-Ras(G12D) block its interaction with Raf. ACS Cent. Sci. 6, 1753-1761 (2020).

22. Fell, J. B. et al. Discovery of tetrahydropyridopyrimidines as irreversible covalent inhibitors of KRAS-G12C with in vivo activity. ACS Med. Chem. Lett. 9, 1230-1234 (2018).

23. Su, H. \& Xu, Y. Application of ITC-based characterization of thermodynamic and kinetic association of ligands with proteins in drug design. Front. Pharmacol. 9, 1133 (2018).

24. Holdgate, G. A. \& Ward, W. H. J. Measurements of binding thermodynamics in drug discovery. Drug Discov. Today 10, 1543-1550 (2005).

25. Trahey, M. \& Mccormick, F. A cytoplasmic protein stimulates normal N-Ras p21 GTPase, but does not affect oncogenic mutants. Science 238, 542-545 (1987).

26. Gentile, D. R. et al. Ras binder induces a modified switch-ll pocket in GTP and GDP States. Cell Chem. Biol. 24, 1455-1466 (2017).

27. Zeng, M. et al. Potent and selective covalent quinazoline inhibitors of KRAS G12C. Cell Chem. Biol. 24, 1005-1016 (2017).

28. Mortier, J. et al. Computationally empowered workflow identifies novel covalent allosteric binders for KRAS(G12C). ChemMedChem 15, 827-832 (2020).

29. McGregor, L. M., Jenkins, M. L., Kerwin, C., Burke, J. E. \& Shokat, K. M. Expanding the scope of electrophiles capable of targeting K-Ras oncogenes. Biochemistry 56, 3178-3183 (2017)

30. Lu, J. et al. KRAS G12C drug development: Discrimination between switch-ll pocket configurations using hydrogen/deuterium-exchange mass spectrometry. Structure 25, 1442-1448 (2017).

31. Weber, C. K., Slupsky, J. R., Kalmes, H. A. \& Rapp, U. R. Active Ras induces heterodimerization of cRaf and BRaf. Cancer Res. 61, 3595-3598 (2001).

32. Karreth, F. A., Frese, K. K., DeNicola, G. M., Baccarini, M. \& Tuveson, D. A. C-Raf is required for the initiation of lung cancer by K-Ras(G12D). Cancer Discov. 1, 128-136 (2011).

33. Blasco, R. B. et al. c-Raf, but not B-Raf, is essential for development of K-Ras oncogene-driven non-small cell lung carcinoma. Cancer Cell 19, 652-663 (2011).

34. Hingorani, S. R. et al. Preinvasive and invasive ductal pancreatic cancer and its early detection in the mouse. Cancer Cell 4, 437-450 (2003).

35. Gray, J. L. et al. Targeting the small GTPase superfamily through their regulatory proteins. Angew. Chem. Int. Ed. Engl. 59, 6342-6366 (2020).

36. Dias Carvalho, P. et al. KRAS oncogenic signaling extends beyond cancer cells to orchestrate the microenvironment. Cancer Res. 78, 7-14 (2018).

37. Dey, P. et al. Oncogenic KRAS-driven metabolic reprogramming in pancreatic cancer cells utilizes cytokines from the tumor microenvironment. Cancer Discov. 10, 608-625 (2020).

38. Liou, G. Y. et al. Mutant KRAS-induced expression of ICAM-1 in pancreatic acinar cells causes attraction of macrophages to expedite the formation of precancerous lesions. Cancer Discov. 5, 52-63 (2015).

39. Ischenko, I. et al. KRAS drives immune evasion in a genetic model of pancreatic cancer. Nat. Commun. 12, 1482 (2021).

40. Kawakami, Y. et al. Immune-resistant mechanisms in cancer immunotherapy. Int. J. Clin. Oncol. 25, 810-817 (2020).

41. Emsley, P. \& Cowtan, K. Coot: model-building tools for molecular graphics. Acta Crystallogr. D. Biol. Crystallogr. 60, 2126-2132 (2004).

42. Maurer, T. et al. Small-molecule ligands bind to a distinct pocket in Ras and inhibit SOS-mediated nucleotide exchange activity. Proc. Natl. Acad. Sci. USA 109, 5299-5304 (2012).

43. Burns, M. C. et al. Approach for targeting Ras with small molecules that activate SOS-mediated nucleotide exchange. Proc. Natl. Acad. Sci. USA 111, 3401-3406 (2014). 